\title{
Principes locaux-globaux pour certaines fibrations en torseurs sous un tore
}

\author{
BY ARNE SMEETS \\ Departement Wiskunde, KU Leuven, Leuven, Belgium, and \\ Département de Mathématiques, Université Paris-Sud 11, Orsay, France. \\ e-mail: arnesmeets@gmail.com
}

(Received 24 July 2013; Revised copy received 23 September 2013)

\begin{abstract}
Let $k$ be a number field and $T$ a $k$-torus. Consider a family of torsors under $T$, i.e. a morphism $f: X \rightarrow \mathbb{P}_{k}^{1}$ from a projective, smooth $k$-variety $X$ to $\mathbb{P}_{k}^{1}$, the generic fibre $X_{\eta} \rightarrow \eta$ of which is a smooth compactification of a principal homogeneous space under $T \otimes_{k} \eta$. We study the Brauer-Manin obstruction to the Hasse principle and to weak approximation for $X$, assuming Schinzel's hypothesis. We generalise Wei's recent results [21]. Our results are unconditional if $k=\mathbf{Q}$ and all non-split fibres of $f$ are defined over $\mathbf{Q}$. We also establish an unconditional analogue of our main result for zero-cycles of degree 1.
\end{abstract}

\section{Résumé}

Soit $k$ un corps de nombres et soit $T$ un $k$-tore. Considérons une fibration en torseurs sous $T$, c'est-à-dire un morphisme $f: X \rightarrow \mathbb{P}_{k}^{1}$ d'une $k$-variété projective et lisse $X$ vers $\mathbb{P}_{k}^{1}$ tel que sa fibre générique $X_{\eta} \rightarrow \eta$ soit une compactification lisse d'un espace principal homogène sous $T \otimes_{k} \eta$. On étudie dans ce texte l'obstruction de Brauer-Manin au principe de Hasse et à l'approximation faible pour $X$, sous l'hypothèse de Schinzel. On généralise les résultats récents de Wei [21]. Nos résultats sont inconditionnels si $k=\mathbf{Q}$ et les fibres non-scindées de $f$ sont définies sur $\mathbf{Q}$. On établit également un analogue inconditionnel de notre résultat principal pour les zéro-cycles de degré 1 .

\section{Introduction}

\subsection{Historique et résultats}

Fixons un corps de nombres $k$, une clôture algébrique $\bar{k}$ de $k$ et une extension abélienne maximale $k^{\text {ab }} \subseteq \bar{k}$ de $k$. Pour les notions de base sur le principe de Hasse, l'approximation faible, le groupe de Brauer et l'obstruction de Brauer-Manin, voir par exemple $[5, \S 3]$.

On s'intéresse dans cet article aux principes locaux-globaux pour certaines variétés projectives et lisses $X$, définies sur le corps de nombres $k$ et fibrées sur la droite projective, c'est-à-dire munies d'un morphisme (dominant) $f: X \rightarrow \mathbb{P}_{k}^{1}$.

La question si l'obstruction de Brauer-Manin contrôle la validité du principe de Hasse et l'approximation faible pour l'espace total d'une telle fibration $f: X \rightarrow \mathbb{P}_{k}^{1}$, s'il en est ainsi pour les fibres de $f$, a été étudiée par de nombreux auteurs. Citons par exemple les 
articles classiques de Harari [12], Colliot-Thélène et Swinnerton-Dyer [9] et ColliotThélène, Skorobogatov et Swinnerton-Dyer [8]. Ce problème est difficile: l'hypothèse de Schinzel $[9, \S 4]$ est devenu un outil standard pour traiter le cas d'une fibration avec plusieurs fibres non-scindées. Or, si on ne suppose pas que le principe de Hasse et l'approximation faible valent pour les fibres de $f$ (comme dans [8]) mais seulement que l'obstruction de Brauer-Manin est la seule dans les fibres, très peu est connu sur la situation. On s'intéresse dans ce texte à une classe particulière de telles fibrations:

Définition $1 \cdot 1$. Soit $T$ un $k$-tore. On définit une fibration en torseurs sous $T$ comme une $k$-variété projective, lisse et géométriquement connexe $X$, munie d'un morphisme dominant $f: X \rightarrow \mathbb{P}^{1}$ tel que la fibre générique $X_{\eta} \rightarrow \eta$ de $f$ soit une compactification lisse d'un espace principal homogène sous le $\eta$-tore $T \times_{k} \eta$.

Le cas particulier d'un tore dit "normique" $T=R_{K / k}^{1} \mathbb{G}_{m}$ associé à une extension finie $K / k$ a été étudié de façon très intensive. Ce cas correspond à la classe des variétés qui sont des compactifications lisses d'une variété affine donnée par une équation de la forme

$$
N_{K / k}(\mathbf{x})=P(t) \neq 0,
$$

où $P(t) \in k[t]$ est un polynôme non constant. Citons les articles récents $[\mathbf{1}, \mathbf{1 0}, \mathbf{1 5}, \mathbf{1 7}$, 20 et 21]. Le spectre des techniques employées est large: citons la méthode de la descente $([\mathbf{4}, \mathbf{1 0}])$, les techniques de la théorie analytique des nombres $([\mathbf{1}])$, la méthode des fibrations $([15,21])$ ou une combinaison de celles-ci $([4,13,17])$.

On généralise ici les résultats de Wei [21] obtenus via la méthode des fibrations (sous l'hypothèse de Schinzel), tout en donnant des preuves plus simples. Wei a étudié l'obstruction de Brauer-Manin pour les équations "normiques" $N_{K / k}(\mathbf{x})=P(t)$, avec $K / k$ une extension abélienne. Son résultat principal [21, theorem 3.1] dit que le principe de Hasse et l'approximation faible valent pour toute compactification lisse d'une telle variété (sous l'hypothèse de Schinzel), dès que l'obstruction de Brauer-Manin ne s'y oppose pas et que la condition $\mathrm{X}_{\omega}^{2}(\widehat{T})=\mathrm{X}_{\omega}^{2}(\widehat{T})_{P}$ est satisfaite (voir [4, définition 2.4]). Comme il le remarque $[21, \S 3]$, cette dernière condition est équivalente à la surjectivité de la flèche naturelle

$$
\operatorname{Br}\left(X^{\mathrm{CTHS}}\right) / \operatorname{Br}(k) \longrightarrow \operatorname{Br}\left(X_{\eta}\right) / \mathrm{Br}(\eta),
$$

où $X^{\mathrm{CTHS}}$ est une certaine compactification partielle de la variété affine définie par l'équation $N_{K / k}(\mathbf{x})=P(t) \neq 0$, construite dans $[4, \S 2]$.

On généralise son résultat dans plusieurs directions, dans le sens qu'on traite des tores généraux au lieu des tores "normiques", et qu'on impose une condition moins forte que sa condition $\mathrm{X}_{\omega}^{2}(\widehat{T})=\mathrm{X}_{\omega}^{2}(\widehat{T})_{P}$.

Le résultat principal de ce texte - qui englobe également [21, theorem 3.2] - est le théorème suivant.

ThÉORÈme 1-2. Soit $T$ un k-tore quasi-déployé par $k^{\mathrm{ab}}$. Soit $f: X \rightarrow \mathbb{P}_{k}^{1}$ une fibration en torseurs sous $T$. Alors le groupe $\operatorname{Br}\left(X_{\eta}\right) / \operatorname{Br}(\eta)$ est abélien fini. Faisons l'hypothèse qu'il existe un ouvert $X^{\circ} \subseteq X$ lisse, surjectif et génériquement projectif à fibres connexes audessus de $\mathbb{A}_{k}^{1}$, de façon que le morphisme $X^{\circ} \rightarrow \mathbb{A}_{k}^{1}$ admette une section sur $k^{\mathrm{ab}}$ et que $\operatorname{Br}\left(X_{\eta}\right) / \operatorname{Br}(\eta)$ soit engendré par les images des groupes suivants:

(i) le groupe $\operatorname{Br}\left(X^{\circ}\right)$, et

(ii) le groupe $\operatorname{Ker}\left[\operatorname{Br}\left(X_{\eta}\right) \rightarrow \operatorname{Br}\left(X_{\eta} \otimes_{k} k^{\mathrm{ab}}\right)\right]$.

Admettons l'hypothèse de Schinzel. Alors $X(k)$ est dense dans $X\left(\mathbf{A}_{k}\right)^{\operatorname{Br}(X)}$. 
Rappelons qu'une extension de corps $K / k$ quasi-déploie le tore $T$ si le $K$-tore $T \otimes_{k} K$ est quasi-trivial, i.e. produit de facteurs de la forme $R_{L / K} \mathbb{G}_{m, L}$, pour $L / K$ une extension finie (et variable).

Remarque 1.3. Si $k=\mathbf{Q}$ et si toutes les fibres non-scindées de $f-\operatorname{voir}[\mathbf{1 8}, \S 1]$ pour cette notion - sont définies sur $\mathbf{Q}$, on peut démontrer le Théorème 1.2 de façon inconditionnelle, c'est-à-dire sans admettre l'hypothèse de Schinzel. On verra ci-dessous que cela résulte des travaux récents de Green, Tao et Ziegler en combinatoire additive, dans la forme présentée dans [14]. Au $§ 5$, on établira l'analogue inconditionnel du Théorème 1.2 pour les zéro-cycles de degré 1 (voir Théorème 5·1).

L'hypothèse faite sur le groupe de Brauer de la fibre générique dans l'énoncé principal est quelque peu technique; il n'est pas évident de la vérifier dans des cas concrets. Or, il se trouve que cette hypothèse est toujours satisfaite si on suppose qu'il existe une extension cyclique de $k$ qui quasi-déploie le tore $T$. Ceci donne le corollaire suivant, qui sera démontré à la fin du $\$ 4$ :

COROllaire 1.4. Soit $T$ un $k$-tore qui est quasi-déployé par une extension cyclique. Soit $f: X \rightarrow \mathbb{P}_{k}^{1}$ une fibration en torseurs sous T. Admettons l'hypothèse de Schinzel. Alors $X(k)$ est dense dans $X\left(\mathbf{A}_{k}\right)^{\operatorname{Br}(X)}$.

En particulier:

COROllaire 1.5. Soit X un modèle projectif et lisse de la $k$-variété affine définie par

$$
\prod_{i=1}^{n} N_{K_{i} / k}\left(\mathbf{x}_{i}\right)=P(t) \neq 0,
$$

où $P(t) \in k[t]$ est quelconque et les corps $K_{1}, \ldots, K_{n}$ sont des extensions finies $d u$ corps de nombres $k$ dont au moins une est une extension cyclique. Admettons l'hypothèse de Schinzel. Alors $X(k)$ est dense dans $X\left(\mathbf{A}_{k}\right)^{\mathrm{Br}(X)}$.

Si $k=\mathbf{Q}$ et si le polynôme $P(t)$ a toutes ses racines rationnelles, le résultat ci-dessus est inconditionnel, comme on l'a remarqué plus haut.

Finalement, au $\S 6$ (qui est indépendant du reste du texte), on fait une remarque sur l'hypothèse de Schinzel qui nous permet de l'utiliser de façon plus souple. Ceci mènera à une généralisation de $[\mathbf{8}$, theorem $1 \cdot 1 \cdot \mathrm{e}]$.

\section{Généralités sur les fibrations en torseurs sous un tore}

Soit $k$ un corps de caractéristique zéro $k$ et soit $T$ un $k$-tore quelconque. Choisissons une compactification lisse et équivariante $T^{c}$ de $T$.

Soit $f: X \rightarrow \mathbb{P}_{k}^{1}$ une fibration en torseurs sous le tore $T$. Il existe alors un ouvert $U \subseteq \mathbb{P}_{k}^{1}$ et un ouvert $V \subseteq X_{U}=f^{-1}(U)$ de façon que $V$ soit un espace principal homogène sur $U$ sous $T$. Le produit contracté $V \times{ }^{T} T^{c}$ est alors une $k$-variété projective et lisse au-dessus de $U$ qui contient $V$ comme ouvert. Des arguments classiques impliquent que le morphisme $V \times^{T} T^{c} \rightarrow U$ s'étend en un morphisme $g: Y \rightarrow \mathbb{P}_{k}^{1}$ qui satisfait $g^{-1}(U)=V \times^{T} T^{c}$, où $Y$ est une $k$-variété projective et lisse $k$-birationnelle à $X$.

Or, les énoncés que l'on veut établir dans ce texte ne dépendent que de la classe d'équivalence birationnelle de la variété projective et lisse $X$. On suppose donc jusqu'à la fin du $\S 3$ que la $k$-variété $X$ est une fibration en torseurs sous $T$ telle qu'au-dessus d'un 
ouvert convenable $U \subseteq \mathbb{P}_{k}^{1}$, l'image inverse $X_{U}=f^{-1}(U)$ est de la forme $V \times{ }^{T} T^{c}$, où $V$ est un $U$-torseur sous $T(\star)$.

Lemme 2.1. Soit $k$ un corps de nombres. Pour tout $Q \in U(k)$, on dispose d'une flèche de spécialisation

$$
\operatorname{Br}\left(X_{\eta}\right) / \operatorname{Br}(\eta) \longrightarrow \operatorname{Br}\left(X_{Q}\right) / \operatorname{Br}(k)
$$

qui est un isomorphisme.

Démonstration. On fixe un point à l'infini $\infty \in \mathbf{P}_{k}^{1}$. Soit $t$ une coordonnée affine sur Spec $k[t]=\mathbf{A}_{k}^{1}=\mathbf{P}_{k}^{1} \backslash\{\infty\}$. Soit $R=\operatorname{Spec} \bar{k}[t]_{(t-\lambda)}$, où $\lambda$ est la $t$-coordonnée de $Q \in U(k)$. Considérons le $R$-schéma $X_{R}$; sa fibre générique est $X_{\eta} \otimes_{k} \bar{k}$. Par [12, lemme 3·1·1], on a un isomorphisme

$$
\operatorname{Pic}\left(X_{R}\right) \cong \operatorname{Pic}\left(X_{\eta} \otimes_{k} \bar{k}\right) .
$$

La fibre spéciale de $X_{R}$ est $\overline{X_{Q}}$; on obtient donc une flèche

$$
\operatorname{Pic}\left(X_{\eta} \otimes_{k} \bar{k}\right) \longrightarrow \operatorname{Pic}\left(\overline{X_{Q}}\right)
$$

qui est un isomorphisme Galois-équivariant [4, lemme 2·1]. Il en résulte que

$$
H^{1}\left(k, \operatorname{Pic}\left(X_{\eta} \otimes_{k} \bar{k}\right)\right) \cong H^{1}\left(k, \operatorname{Pic}\left(\bar{X}_{Q}\right)\right) \cong \operatorname{Br}\left(X_{Q}\right) / \operatorname{Br}(k) .
$$

Pour finir la preuve, il suffit maintenant de remarquer que

$$
H^{1}\left(k, \operatorname{Pic}\left(X_{\eta} \otimes_{k} \bar{k}\right)\right) \cong H^{1}\left(\eta, \operatorname{Pic}\left(X_{\bar{\eta}}\right)\right) \cong \operatorname{Br}\left(X_{\eta}\right) / \operatorname{Br}(\eta) .
$$

(Notons que ce dernier énoncé utilise $H^{3}\left(k(t), \mathbb{G}_{m}\right)=0$, pour $k$ un corps de nombres.)

La proposition suivante se montre utile pour l'étude des fibres non scindées d'une fibration en torseurs sous un tore. Il implique en particulier que si le $k$-tore $T$ est déployé (ou quasi-déployé) par une extension abélienne de $k$, alors la fibration satisfait à la condition d'abélianité de [8, theorem 1·1]. Pour la définition de tores flasques, on renvoie à [6, definition 1.2].

PROPOSITION 2-2. On suppose qu'il existe une extension finie $K$ du corps $k$ telle que le $K$-tore $T \otimes_{k} K$ soit flasque. Soit $Q \in \mathbb{P}_{k}^{1}$ un point fermé de corps résiduel $L$.

Alors $X_{Q}$ admet une composante irréductible $Y$ de multiplicité 1 telle que la fermeture algébrique de $L$ dans le corps de fonctions $L(Y)$ soit un sous-corps d'un corps qui est facteur direct de $K \otimes_{k} L$.

Démonstration. La $K$-variété $X_{U} \otimes_{k} K$ est un torseur sur $U \otimes_{k} K$ sous $T \otimes_{k} K$ et s'étend en un torseur sur $\mathbb{P}_{K}^{1}$ tout entier grâce à [6, theorem 2.2.(i)]. Si $R$ est un point fermé de $\mathbb{P}_{K}^{1}$ au-dessus de $Q$, alors [3, proposition 3.4.(b)] implique que la fibre $\left(X \otimes_{k} K\right)_{R}$ a une composante géométriquement intègre de multiplicité 1 . On en déduit facilement le résultat voulu.

Soit $X^{\circ} \subseteq X$ un ouvert lisse, surjectif et génériquement projectif à fibres connexes sur la droite affine $\mathbb{A}_{k}^{1}$, tel que le morphisme induit $X^{\circ} \rightarrow \mathbb{A}_{k}^{1}$ admet une section sur $\bar{k}$. Un tel ouvert $X^{\circ}$ existe toujours: si $L$ est une extension de $k$ qui déploie le tore $T$, alors la fibre générique $X_{\eta} \otimes_{k} L$ est un torseur sous le tore déployé $\left(T \times_{k} \eta\right) \otimes_{k} L$. Comme tout espace principal homogène sous un tore trivial est trivial, on voit que $X_{\eta} \otimes_{k} L$ admet un $\left(\eta \otimes_{k} L\right)$-point qui s'étend en une section $\mathbb{P}_{L}^{1} \rightarrow X \otimes_{k} L$ dont l'image est contenue dans le lieu lisse de $X$. 
Pour $X^{\circ}$ on prend alors un ouvert approprié - c'est-à-dire lisse, surjectif et génériquement projectif à fibres connexes sur la droite affine $\mathbb{A}_{k}^{1}$ - qui contient l'image de $\mathbb{A}_{L}^{1}$ par cette section.

On remarque qu'il suffit (dans le raisonnement ci-dessus) de prendre une extension $L$ qui quasi-déploie le tore $T$, comme tout torseur sous un tore quasi-trivial est trivial.

Lemme 2.3. Soit $K$ une extension finie de $k$ qui quasi-déploie le tore $T$, et telle que le morphisme $p: X^{\circ} \rightarrow \mathbb{A}_{k}^{1}$ admet une section $\sigma$ sur K. Alors on a

$$
\mathrm{Br} K \stackrel{\sim}{\longrightarrow} \mathrm{Br} X_{K}^{\circ} \text {. }
$$

Démonstration. La fibre générique $X_{\eta}^{\circ}$ du morphisme $X^{\circ} \rightarrow \mathbb{A}_{k}^{1}$ est une $\eta$-variété projective, lisse et géométriquement intègre. La variété $X_{\eta \otimes_{k} K}$ est rationnelle sur $\eta \otimes_{k} K-$ l'extension $K$ de $k$ quasi-déploie le tore $T$ et tout torseur sous un tore quasi-trivial est trivial. Il en résulte donc que $\operatorname{Br} X_{\eta \otimes_{k} K}=\operatorname{Br}\left(\eta \otimes_{k} K\right)$, car le groupe de Brauer est un invariant birationnel des variétés projectives lisses (sur un corps de caractéristique zéro). D'après Grothendieck, on dispose donc d'une injection $\operatorname{Br} X_{K}^{\circ} \hookrightarrow \operatorname{Br}\left(X_{\eta_{\otimes_{k} K}}\right)$ qui s'insère dans le diagramme commutatif suivant:

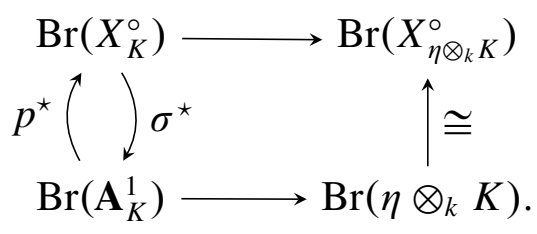

Il est évident que $\sigma^{\star} \circ p^{\star}=$ Id; en particulier, l'homomorphisme $\sigma^{\star}$ est surjectif. La commutativité du diagramme implique que la flèche composée

$$
\operatorname{Br}\left(X_{K}^{\circ}\right) \stackrel{\sigma^{\star}}{\longrightarrow} \operatorname{Br}\left(\mathbb{A}_{K}^{1}\right) \longrightarrow \operatorname{Br}\left(\eta \otimes_{k} K\right)
$$

est injective. Il en résulte que $\sigma^{\star}$ est injectif, donc un isomorphisme.

Comme $\operatorname{Br}\left(\mathbb{A}_{K}^{1}\right)=\operatorname{Br}(K)$, ceci permet de conclure.

\section{Preuve du résultat principal}

\subsection{La preuve}

Ce paragraphe est consacré à la preuve du Théorème 1.2. On utilise les notations introduites dans le paragraphe précédent et l'on suppose désormais que $k$ est un corps de nombres. Choisissons

$$
\alpha_{1}, \ldots, \alpha_{m} \in \operatorname{Br}\left(X^{\circ}\right) \text { et } \beta_{1}, \ldots, \beta_{n} \in \operatorname{Ker}\left[\operatorname{Br}\left(X_{\eta}\right) \longrightarrow \operatorname{Br}\left(X_{\eta} \otimes_{k} k^{\mathrm{ab}}\right)\right]
$$

tels que leurs images engendrent $\operatorname{Br}\left(X_{\eta}\right) / \operatorname{Br}(\eta)$. Ce dernier quotient est un groupe abélien fini, la raison étant que la $\eta$-variété $X_{\eta}$ et projective, lisse, géométriquement connexe et géométriquement rationnelle. Fixons une extension finie abélienne $M$ de $k$ qui quasi-déploie le tore $T$, pour laquelle le morphisme $X^{\circ} \rightarrow \mathbb{A}_{k}^{1}$ admet une section après extension des scalaires à $M$ et telle que

$$
\operatorname{Res}_{k / M}\left(\beta_{i}\right)=0 \text { pour } 1 \leqslant i \leqslant n .
$$


On suppose qu'il n'y ait pas d'obstruction de Brauer-Manin au principe de Hasse et à l'approximation faible, c'est-à-dire que l'égalité

$$
\sum_{v \in \Omega_{k}} \operatorname{inv}_{v}\left(\mathcal{A}\left(P_{v}\right)\right)=0
$$

vaut pour tout $\alpha \in \operatorname{Br}(X)$ et tout point adélique $\left(P_{v}\right)_{v \in \Omega_{k}} \in X\left(\mathbf{A}_{k}\right)$. On fixe un tel point $\left(P_{v}\right)_{v \in \Omega_{k}} \in X\left(\mathbf{A}_{k}\right)$ et un ensemble fini $S$ de places de $k$. On démontrera que l'on peut approximer les points locaux $\left(P_{v}\right)_{v \in S}$ par un point global $P \in X(k)$.

En cours de route, on peut toujours agrandir l'ensemble fini de places $S$. On peut donc supposer sans perte de généralité que $S$ contient toutes les places archimédiennes de $k$. Considérons le sous-groupe $B=\left\langle\alpha_{1}, \ldots, \alpha_{m}, \beta_{1}, \ldots, \beta_{n}\right\rangle$ de $\operatorname{Br}\left(X_{\eta}\right)$. Un argument de passage à la limite en cohomologie étale permet de supposer $B \subseteq \operatorname{Br}\left(X_{U}\right)$, en rétrécissant l'ouvert $U$ si nécessaire. En agrandissant l'ensemble $S$ si nécessaire, on peut supposer que les morphismes $X_{U} \rightarrow U \rightarrow k$ admettent des modèles entiers $\mathcal{X}_{\mathcal{U}} \rightarrow \mathcal{U} \rightarrow \mathcal{O}_{k, S}$ tels que les fibres de $\mathcal{X}_{\mathcal{U}} \rightarrow \mathcal{U}$ soient toutes projectives, lisses et géométriquement intègres, de façon que $X^{\circ} \rightarrow \mathbb{A}_{k}^{1}$ s'étende en un morphisme $\mathcal{X}^{\circ} \rightarrow \mathbb{A}_{\mathcal{O}_{k, S}}^{1}$. En agrandissant encore $S$, on peut alors supposer que les éléments de $B$ appartiennent tous au sous-groupe $\operatorname{Br}\left(\mathcal{X}_{\mathcal{U}}\right)$ de $\operatorname{Br}\left(X_{\eta}\right)$. Le lemme de Hensel et les estimations de Lang-Weil permettent de supposer également que pour tout $v \notin S$ et $\mathcal{Q}$ point fermé de $\mathcal{U}$, la fibre $\left(\mathcal{X}_{\mathcal{U}}\right)_{\mathcal{Q}}$ a un $\kappa(v)$-point $(\kappa(v)$ étant le corps résiduel associé à $v$ ).

Comme $X$ est lisse (et géométriquement intègre) sur $k$, le théorème des fonctions implicites assure que tout voisinage $v$-adique de $P_{v}$ est Zariski dense dans $X$. On peut donc remplacer chaque $P_{v}$ par un $k_{v}$-point arbitrairement proche, que l'on notera encore $P_{v}$, qui appartient à $X_{U}$. Notons $Q_{v}=p\left(P_{v}\right)$ pour tout $v \in S$.

Choisissons (en utilisant l'approximation faible) $Q_{0} \in U(k)$, suffisamment proche de $Q_{v}$ pour $v \in S$ archimédienne, différent des points $Q_{v}$. En utilisant un $k$-automorphisme convenable de $\mathbb{P}_{k}^{1}$, on peut supposer que $Q_{0}$ est le point à l'infini. Les points $\left(P_{v}\right)_{v \in S}$ appartiennent donc à $\mathbb{A}_{k}^{1}=\operatorname{Spec}(k[t]) \subseteq \mathbb{P}_{k}^{1}$. Notons alors $\lambda_{v} \in k_{v}$ pour la $t$-coordonnée de $Q_{v} \in \mathbb{A}^{1}\left(k_{v}\right)$. On cherche $Q \in U(k)$ très proche de $Q_{v}$ pour toute place finie $v \in S$ et suffisamment grand pour toute place archimédienne tel que la fibre $X_{Q}$ ait un point rationnel, très proche des points locaux $P_{v}$ pour $v \in S$.

Le complément de $U$ dans $\mathbb{A}_{k}^{1}$ est réunion de points $Q_{i} \in \mathbb{A}_{k}^{1}=\operatorname{Spec}(k[t])$, décrits par des polynômes unitaires irréductibles $F_{i}(t) \in k[t]$, pour $1 \leqslant i \leqslant s$. Considérons tous les éléments de $\operatorname{Br}(k(X))$ de la forme $\left(F_{i}(t), \chi\right)$, où $\chi$ est un caractère de $M / k$. Ceux-ci sont définis par cup-produit

$$
k(X)^{\star} \times H^{2}(k, \mathbf{Z}) \longrightarrow \operatorname{Br}(k(X)),
$$

via l'identification $H^{1}(k, \mathbf{Q} / \mathbf{Z}) \cong H^{2}(k, \mathbf{Z})$. Il est clair que ces éléments s'étendent en des algèbres d'Azumaya sur $X_{U}$. Soit $B^{\prime}$ le sous-groupe fini de $\operatorname{Br}\left(X_{U}\right)$ engendré par ces éléments et ceux de $B$. En agrandissant $S$, on peut supposer que les éléments $\left(F_{i}(t), \chi\right)$ appartiennent à $\operatorname{Br}\left(\mathcal{X}_{\mathcal{U}}\right)$, i.e. $B^{\prime} \subseteq \operatorname{Br}\left(\mathcal{X}_{\mathcal{U}}\right)$.

Agrandissons encore l'ensemble $S$ : on ajoute les places associées aux polynômes $F_{i}(t)$ via l'hypothèse $\left(\mathrm{H}_{1}\right)$ [9] et les places qui ramifient dans l'extension $M / k$. Considérons l'image de $\operatorname{Br}\left(X^{\circ}\right)$ dans $\operatorname{Br}\left(X_{M}^{\circ}\right)$. Le Lemme 2.3 implique que pour tout $i$, on peut trouver un élément $\gamma_{i} \in \operatorname{Br} M$ tel que $\gamma_{i}$ s'envoie sur l'image de $\alpha_{i}$ via la flèche canonique $\operatorname{Br} M \rightarrow \operatorname{Br}\left(X_{M}^{\circ}\right)$. En prenant $S$ suffisamment grand, on peut maintenant supposer que $\operatorname{inv}_{w} \gamma_{i}=0$ pour 
$i \in\{1, \ldots, m\}$ et pour $w \notin S_{M}$ ( $S_{M}$ étant l'ensemble des places de $M$ au-dessus des places dans $S$ ).

On suppose qu'il n'y a pas d'obstruction de Brauer-Manin à l'approximation faible pour $\left(P_{v}\right)_{v \in S}$. Le lemme formel de Harari ([12, corollaire 2.6.1]) donne alors l'existence d'un ensemble fini de places $S_{1} \supseteq S$ et de points locaux $P_{v} \in X_{U}\left(k_{v}\right)$ pour $v \in S_{1} \backslash S$, tels que pour tout $\mathcal{A} \in B^{\prime}$,

$$
\sum_{v \in S_{1}} \operatorname{inv}_{v}\left(\mathcal{A}\left(P_{v}\right)\right)=0 \in \mathbf{Q} / \mathbf{Z} .
$$

L'hypothèse $\left(\mathrm{H}_{1}\right)$ donne alors l'existence de $\lambda \in k$ qui est suffisamment proche de $\lambda_{v}$ pour toute place finie $v \in S_{1}$, entier en dehors de $S_{1}$ et suffisamment grand pour les places archimédiennes, tel qu'on ait (simultanément):

(i) $\lambda \in U(k)$;

(ii) pour $v \in S_{1}$, la fibre $X_{\lambda}$ contient un $k_{v}$-point $P_{v}^{\prime}$ qui est $v$-adiquement proche de $P_{v}$, tel que $\operatorname{inv}_{v}\left(\mathcal{A}\left(P_{v}\right)\right)=\operatorname{inv}_{v}\left(\mathcal{A}\left(P_{v}^{\prime}\right)\right)$ pour tout $\mathcal{A} \in B^{\prime}$;

(iii) pour $1 \leqslant i \leqslant s$, il existe une place $v_{i}$ de $k$ telle que $F_{i}(\lambda)$ est une uniformisante dans $\mathcal{O}_{k, v_{i}}$ et une unité dans $k_{v}$ pour toute $v \notin S_{1} \cup\left\{v_{i}\right\}$.

Pour $v \notin S_{1}$ et $v \neq v_{i}$ pour tout $i$, on obtient alors, grâce au lemme de Hensel, un $\mathcal{O}_{v}$-point de la fibre $\left(\mathcal{X}_{\mathcal{U}}\right)_{\lambda}$. L'ensemble $S$ a été pris suffisamment grand pour que $B^{\prime} \subseteq \operatorname{Br}\left(\mathcal{X}_{\mathcal{U}}\right)$. On obtient donc de cette façon un $\mathcal{O}_{v}$-point $P_{v}^{\prime}$ de $X_{\lambda}$ tel que $\operatorname{inv}_{v} \alpha\left(P_{v}^{\prime}\right)=0$ pour tout $\alpha \in B^{\prime}$ ("bonne réduction").

Analysons maintenant la situation si $v=v_{i}$ est l'une des "places de Schinzel". Notons que par la loi de réciprocité globale, on a, pour tout caractère $\chi$ de $M / k$,

$$
\sum_{v \in \Omega_{k}} \operatorname{inv}_{v}\left(F_{i}(\lambda), \chi\right)=0 .
$$

Par construction, on a également

$$
\sum_{v \in S_{1}} \operatorname{inv}_{v}\left(F_{i}(\lambda), \chi\right)=0 .
$$

Pour $v \notin S_{1}$ et $v \neq v_{i}$, on sait que $F_{i}(\lambda)$ est une unité dans $k_{v}$. On trouve donc

$$
\operatorname{inv}_{v_{i}}\left(F_{i}(\lambda), \chi\right)=0 .
$$

Cette dernière égalité vaut pour tout caractère $\chi$ de $M / k$. Il en résulte que la place $v_{i}$ est totalement décomposée dans l'extension (abélienne!) $M / k$, car (par construction) $F_{i}(\lambda)$ est une uniformisante dans $\mathcal{O}_{k, v_{i}}$.

La fibre $X_{\lambda}$ est une compactification lisse d'un espace principal homogène sous $T$, qui devient quasi-trivial après extension des scalaires à $M / k$. Il en résulte que le $k_{v_{i}}$-tore $T_{k_{v_{i}}}$ est quasi-trivial, donc $H^{1}\left(k_{v_{i}}, T_{k_{v_{i}}}\right)=0$. Ceci implique la $k_{v_{i}}$-rationalité de $X_{\lambda} \otimes_{k} k_{v_{i}}$. Il existe donc un $k_{v_{i}}$-point $P_{v_{i}}^{\prime}$.

Montrons que $\operatorname{inv}_{v_{i}} \mathcal{A}\left(P_{v_{i}}^{\prime}\right)=0$ pour $\mathcal{A} \in B$. Comme $v_{i}$ est totalement décomposée dans $M / k$, la flèche $\left\langle\alpha_{1}, \ldots, \alpha_{m}\right\rangle \rightarrow \operatorname{Br}\left(X_{k_{v_{i}}}^{\circ}\right)$ se factorise en

$$
\left\langle\alpha_{1}, \ldots, \alpha_{m}\right\rangle \longrightarrow \operatorname{Br}\left(X_{M}^{\circ}\right) \longrightarrow \operatorname{Br}\left(X_{k_{v_{i}}}^{\circ}\right) .
$$

Pour $1 \leqslant j \leqslant m$, on trouve alors

$$
\alpha_{j}\left(P_{v_{i}}^{\prime}\right)=\left.\alpha_{j}\right|_{k_{v_{i}}}\left(P_{v_{i}}^{\prime}\right)=\left.\gamma_{j}\right|_{k_{v_{i}}}\left(P_{v_{i}}^{\prime}\right)=0 .
$$


Pour $1 \leqslant \ell \leqslant n$, on obtient $\beta_{\ell}\left(P_{v_{i}}^{\prime}\right)=\left.\beta_{\ell}\right|_{k_{v_{i}}}\left(P_{v_{i}}^{\prime}\right)=0$ car $\left.\beta_{\ell}\right|_{M}=0$. Donc

$$
\operatorname{inv}_{v_{i}} \mathcal{A}\left(P_{v_{i}}^{\prime}\right)=0
$$

pour $\mathcal{A} \in B$.

Faisons un résumé de la situation. On a construit pour toute $v \in \Omega_{k}$ un $k_{v}$-point $P_{v}^{\prime}$ de la fibre $X_{\lambda}$. On a $\sum_{v \in S_{1}} \operatorname{inv}_{v} \mathcal{A}\left(P_{v}^{\prime}\right)=0$ et $\operatorname{inv}_{v} \mathcal{A}\left(P_{v}^{\prime}\right)=0$ pour tout $\mathcal{A} \in B$ et $v \notin S_{1}$. On obtient donc $\sum_{v \in \Omega_{k}} \operatorname{inv}_{v} \mathcal{A}\left(P_{v}^{\prime}\right)=0$, i.e. on a construit un point adélique de la fibre $X_{\lambda}$ orthogonal à $B$. Or, le Lemme $2 \cdot 1$ assure que la flèche

$$
\operatorname{Br}\left(X_{\eta}\right) / \operatorname{Br}(\eta) \longrightarrow \operatorname{Br}\left(X_{\lambda}\right) / \operatorname{Br}(k)
$$

est un isomorphisme. L'image du groupe $B$ dans le quotient $\operatorname{Br}\left(X_{\eta}\right) / \operatorname{Br}(\eta)$ engendre celuici. Le point adélique $\left(P_{v}\right)_{v \in \Omega_{k}}$ est donc orthogonal au groupe de Brauer de $X_{\lambda}$, qui est une compactification lisse d'un espace principal homogène sous un $k$-tore. Un résultat classique de Sansuc [16, corollaires 8.7 et 8.13] implique que l'on peut approximer ce point adélique par un point global $P \in X_{\lambda}(k)$, ce qui achève la démonstration.

\subsection{Sur la Remarque $1 \cdot 3$}

Expliquons pourquoi le résultats récents de Green, Tao et Ziegler en combinatoire additive impliquent une version inconditionnelle du Théorème 1.2 si $k=\mathbf{Q}$ et si toutes les fibres non-scindées sont définies sur $\mathbf{Q}$.

Dans la preuve ci-dessus, on peut alors supposer que l'ouvert $U$ est le complément d'un nombre fini de points rationnels. Les polynômes $F_{i}(t)$ dans la preuve sont donc tous de la forme $t-e_{i}$, pour $e_{1}, \ldots, e_{s} \in \mathbf{Q}$. Là où on a choisi $\lambda \in \mathbf{Q}$ utilisant l'hypothèse $\left(\mathrm{H}_{1}\right)$, on peut maintenant choisir $\lambda$ utilisant [14, proposition 2.1]. On trouve donc $\lambda \in \mathbf{Q}$ arbitrairement proche de $\lambda_{v}$ pour $v \in S_{1}$ finie et suffisamment grand pour la topologie réelle, tel que $\lambda$ est une uniformisante dans $\mathbf{Q}_{v_{i}}$ pour $1 \leqslant i \leqslant s$ et

$$
\operatorname{val}_{v}\left(\lambda-e_{i}\right) \leqslant 0 \text { pour } v \notin S_{1} \cup\left\{v_{1}, \ldots, v_{s}\right\} .
$$

Cette dernière inégalité implique que la réduction de $\lambda$ modulo $v$ est un élément de $\mathbb{P}^{1}(\kappa(v))$ autre que les réductions de $e_{1}, \ldots, e_{s}$. Les estimations de Lang-Weil et le lemme de Hensel impliquent que ce $\kappa(v)$-point se relève en un $\mathcal{O}_{v}$-point sur un modèle entier. Le reste de l'argument ne change pas.

\section{Applications}

Comme on l'a déjà expliqué dans l'introduction, notre théorème principal généralise [21, theorem 3·1]. Il englobe également [21, theorem 3·2], qui concerne les modèles projectifs et lisses des variété affines définies par une équation de la forme

$$
\left(X_{1}^{2}-a Y_{1}^{2}\right)\left(X_{2}^{2}-b Y_{2}^{2}\right)\left(X_{3}^{2}-a b Y_{3}^{2}\right)=P(t),
$$

pour $a, b \in k$. Une telle variété est fibrée sur $\mathbb{P}_{k}^{1}$ via la projection sur $t$. Les fibres lisses sont des torseurs sous le tore noyau du morphisme de tores

$$
\begin{aligned}
R_{k(\sqrt{a}) / k} \mathbb{G}_{m} \times R_{k(\sqrt{b}) / k} \mathbb{G}_{m} \times R_{k(\sqrt{a b}) / k} \mathbb{G}_{m} & \longrightarrow \mathbb{G}_{m} \\
(\mathbf{x}, \mathbf{y}, \mathbf{z}) & \longmapsto N_{k(\sqrt{a}) / k}(\mathbf{x}) \cdot N_{k(\sqrt{b}) / k}(\mathbf{y}) \cdot N_{k(\sqrt{a b}) / k}(\mathbf{z}) .
\end{aligned}
$$

Colliot-Thélène a démontré [2, théorème 4.1] que si aucune des constantes $a, b$ et $a b$ n'est un carré dans $k$, alors $\left(X_{1}^{2}-a Y_{1}^{2}, b\right)$ est l'unique élément non-trivial de $\operatorname{Br}\left(X_{\eta}\right) / \operatorname{Br}(\eta)$. 
Comme cet élément de $\operatorname{Br}\left(X_{\eta}\right)$ s'annule après extension des scalaires à l'extension abélienne $k(\sqrt{b}) / k$, le Théorème 1.2 s'applique.

Dans son texte [15], Liang a obtenu des résultats sur les fibrations sous une hypothèse technique (Br) sur le groupe de Brauer (voir par exemple [15, theorem 2.1]) qui paraît moins restrictive que la nôtre. On montre maintenant que ces hypothèses sont en fait équivalentes:

PROPOSITION 4.1. Avec les notations utilisées précédemment au $\S 2$, on considère des points fermés $Q_{1}, Q_{2}, \ldots, Q_{r}$ de $\mathbb{A}_{k}^{1}$ tels que les fibres $Y_{1}, \ldots, Y_{r}$ au-dessus de ces points sont lisses et géométriquement intègres. $\operatorname{Si} \operatorname{Br}\left(X^{\circ} \backslash \bigsqcup_{i=1}^{r} Y_{i}\right)$ engendre $\operatorname{Br}\left(X_{\eta}\right) / \operatorname{Br}(\eta)$, alors $\operatorname{Br}\left(X^{\circ}\right)$ engendre $\operatorname{Br}\left(X_{\eta}\right) / \operatorname{Br}(\eta)$.

Démonstration. Prenons une classe $\bar{\alpha} \in \operatorname{Br} X_{\eta} / \mathrm{Br} \eta$ et $\alpha \in \operatorname{Br}\left(X^{\circ} \backslash \bigsqcup_{i=1}^{r} Y_{i}\right)$ qui s'envoie sur $\bar{\alpha}$. Supposons que $\alpha$ ramifie au point générique de $Y_{i}$. Soit

$$
\partial_{i}: \operatorname{Br}\left(X_{\eta}\right) \longrightarrow H^{1}\left(Y_{i}, \mathbf{Q} / \mathbf{Z}\right)
$$

l'application résidu. On voit facilement que

$$
\operatorname{Br}\left(X_{\eta \otimes_{k} \bar{k}} \cong \operatorname{Br}\left(\eta \otimes_{k} \bar{k}\right)=0\right.
$$

(théorème de Tsen), car $X_{\eta \otimes_{k} \bar{k}}$ est projective, lisse et $\left(\eta \otimes_{k} \bar{k}\right)$-rationnelle.

On a donc le diagramme commutatif suivant:

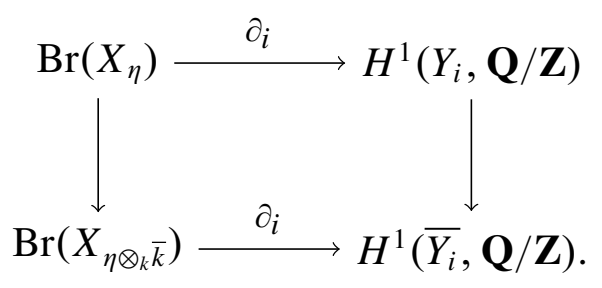

Ceci montre que $\partial_{i}(\alpha)$ appartient au noyau de l'application

$$
H^{1}\left(Y_{i}, \mathbf{Q} / \mathbf{Z}\right) \longrightarrow H^{1}\left(\bar{Y}_{i}, \mathbf{Q} / \mathbf{Z}\right) .
$$

Si $K_{i}=k\left(Q_{i}\right)$ il en résulte que $\partial_{i}(\alpha)$ provient d'un caractère $\chi_{i} \in H^{1}\left(K_{i}, \mathbf{Q} / \mathbf{Z}\right)$ - c'est là que l'on utilise le fait que $Y_{i}$ est géométriquement intègre. Considérons

$$
\beta_{i}:=\operatorname{Cores}_{K_{i} / k}\left(\chi_{i}, t-e_{i}\right) \in \operatorname{Br} \eta,
$$

où $e_{i}$ est la classe de $t$ dans le quotient $K_{i}=k[t] /\left(P_{i}(t)\right)$, où $P_{i}(t)$ est le polynôme qui définit le point fermé $Q_{i}$. Regardons le diagramme commutatif

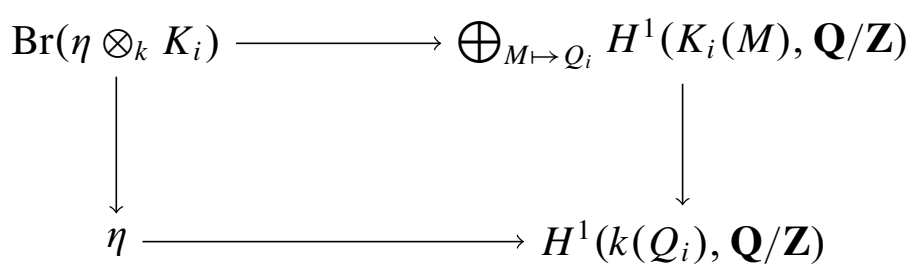

dans lequel les flèches horizontales sont les applications résidu et les flèches verticales sont les applications de corestriction. Ceci montre que $\partial_{Q_{i}}\left(\beta_{i}\right)=\chi_{i}$. L'algèbre $\beta_{i}$ est clairement non-ramifiée en dehors de $Q_{i}$. On voit maintenant que $\alpha-\sum_{i=1}^{r} \beta_{i}$ appartient non seulement à $\operatorname{Br}\left(X^{\circ} \backslash \sqcup Y_{i}\right)$ mais aussi au sous-groupe $\operatorname{Br} X^{\circ}$. Cet élément s'envoie toujours sur $\bar{\alpha} \in \operatorname{Br} X_{\eta} / \mathrm{Br} \eta$. Il en résulte que $\mathrm{Br} X^{\circ} \rightarrow \operatorname{Br} X_{\eta} / \mathrm{Br} \eta$ est surjective. 
Voici un exemple d'application du Théorème 1.2 (cfr. Corollaire 1.4):

Proposition 4-2. Soit $T$ un $k$-tore qui est quasi-déployé par une extension cyclique. Soit $f: X \rightarrow \mathbb{P}_{k}^{1}$ une fibration en torseurs sous T. Admettons l'hypothèse de Schinzel. Alors $X(k)$ est dense dans $X\left(\mathbf{A}_{k}\right)^{\operatorname{Br}(X)}$.

Démonstration. Ceci est une conséquence immédiate du Théorème 1.2 si on vérifie que $\operatorname{Ker}\left[\operatorname{Br}\left(X_{\eta}\right) \rightarrow \operatorname{Br}\left(X_{\eta} \otimes_{k} L\right)\right]$ engendre $\operatorname{Br}\left(X_{\eta}\right) / \operatorname{Br}(\eta)$, où $L / k$ est l'extension cyclique qui quasi-déploie le $k$-tore $T$. Pour l'ouvert $X^{\circ}$ dans le Théorème 1.2, on peut alors prendre n'importe quel ouvert de $X$ qui est lisse, surjectif, génériquement projectif à fibres connexes sur $\mathbb{A}_{k}^{1}$, tel que le morphisme induit $X^{\circ} \rightarrow \mathbb{A}_{k}^{1}$ admette une section sur $L$ (cfr. la discussion qui précède le Lemme 2.3).

Or, ceci résulte facilement de [2, proposition 1·1·(b)], appliquée à $X_{\eta}$. La $\eta$-variété $X_{\eta}$ devient bien rationnelle après extension des scalaires à $L$, car le tore $T \otimes_{k} L$ est quasi-trivial, et tout torseur sous un tore quasi-trivial est trivial.

Ceci donne une généralisation de [21, theorem 3·2]. Comme expliqué ci-dessus, on obtient le même résultat sans admettre l'hypothèse de Schinzel si $k=\mathbf{Q}$ et toutes les fibres nonscindées sont définies sur $\mathbf{Q}$.

\section{Zéro-cycles}

On établit maintenant l'analogue naturel du Théorème 1.2 pour les zéro-cycles de degré 1 au lieu des points rationnels. L'astuce de Salberger servira comme substitut pour l'hypothèse de Schinzel dans notre preuve. On renvoie à $[\mathbf{9}, \S 3.1]$ pour la définition et les propriétés de base de l'accouplement entre le groupe de Brauer d'une variété et les zéro-cycles sur cette variété.

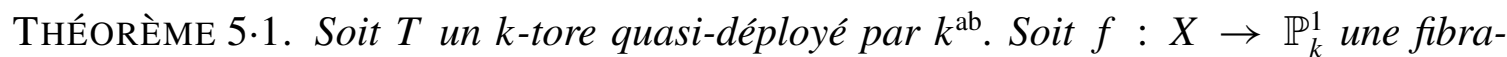
tion en torseurs sous $T$. Supposons qu'il existe un ouvert $X^{\circ} \subseteq X$ qui est lisse, surjectif et génériquement projectif à fibres connexes au-dessus de $\mathbb{A}_{k}^{1}$, de façon que le morphisme $X^{\circ} \rightarrow \mathbb{A}_{k}^{1}$ admette une section sur $k^{\mathrm{ab}}$ et que $\operatorname{Br}\left(X_{\eta}\right) / \operatorname{Br}(\eta)$ soit engendré par les images des groupes suivants:

(i) le groupe $\operatorname{Br}\left(X^{\circ}\right)$, et

(ii) le groupe $\operatorname{Ker}\left[\operatorname{Br}\left(X_{\eta}\right) \rightarrow \operatorname{Br}\left(X_{\eta} \otimes_{k} k^{\mathrm{ab}}\right)\right]$.

Alors l'obstruction de Brauer-Manin à l'existence d'un zéro-cycle de degré 1 sur X est la seule.

Démonstration. On utilise les notations du §2. Choisissons

$$
\alpha_{1}, \ldots, \alpha_{m} \in \operatorname{Br}\left(X^{\circ}\right) \text { et } \beta_{1}, \ldots, \beta_{n} \in \operatorname{Ker}\left[\operatorname{Br}\left(X_{\eta}\right) \longrightarrow \operatorname{Br}\left(X_{\eta} \otimes_{k} k^{\mathrm{ab}}\right)\right]
$$

et une extension abélienne $M$ de $k$ comme dans la preuve du Théorème 1·2. Soit $B$ le sousgroupe $\left\langle\alpha_{1}, \ldots, \alpha_{m}, \beta_{1}, \ldots, \beta_{n}\right\rangle \operatorname{de} \operatorname{Br}\left(X_{\eta}\right)$. En rétrécissant l'ouvert $U$ si nécessaire, on peut supposer sans perte de généralité que $B \subseteq \operatorname{Br}\left(X_{U}\right)$. Le complément de $U$ dans $\mathbb{A}_{k}^{1}$ est réunion de points fermés $Q_{i} \in \mathbb{A}_{k}^{1}=\operatorname{Spec}(k[t])$, décrits par des polynômes unitaires irréductibles $F_{i}(t) \in k[t]$, pour $1 \leqslant i \leqslant s$.

Fixons d'abord un ensemble fini de places $S_{0} \subseteq \Omega_{k}$ tel que les conditions suivantes soient satisfaites. Les morphismes $X_{U} \rightarrow U \rightarrow k$ admettent des modèles $\mathcal{X}_{\mathcal{U}} \rightarrow \mathcal{U} \rightarrow \mathcal{O}_{k, S}$ tels 
que les fibres de $\mathcal{X}_{\mathcal{U}} \rightarrow \mathcal{U}$ soient projectives, lisses et géométriquement intègres, de façon que $X^{\circ} \rightarrow \mathbb{A}_{k}^{1}$ s'étende en un morphisme $\mathcal{X}^{\circ} \rightarrow \mathbb{A}_{\mathcal{O}_{k, S_{0}}}^{1}$. On a des inclusions

$$
B \subseteq \operatorname{Br}\left(\mathcal{X}_{\mathcal{U}}\right) \subseteq \operatorname{Br}\left(X_{\eta}\right)
$$

Pour $v \notin S_{0}$ et $\mathcal{Q}$ point fermé de $\mathcal{U}$, la fibre $\left(\mathcal{X}_{\mathcal{U}}\right)_{\mathcal{Q}}$ a un $\kappa(v)$-point. Pour $v \notin S_{0}$, l'extension $M / k$ est non-ramifiée en $v$. Finalement, pour $\mathcal{A} \in B \cap \operatorname{Br}\left(X^{\circ}\right)$, pour $w$ une place de $M$ non au-dessus d'une place de $S_{0}$ et

$$
j: \operatorname{Br}\left(X^{\circ}\right) \longrightarrow \operatorname{Br}\left(X_{M}^{\circ}\right)=\mathrm{Br}_{0}\left(X_{M}^{\circ}\right)
$$

la flèche naturelle, on $\operatorname{ainv}_{w}(j(\mathcal{A}))=0$.

Soit $B^{\prime}$ le sous-groupe de $\operatorname{Br}\left(\mathcal{X}_{\mathcal{U}}\right)$ engendré par les éléments de $B$ et ceux de la forme $\left(F_{i}(t), \chi\right)$, pour $\chi$ un caractère de l'extension $M / k$. Une variante du lemme formel de Harari (voir [9, theorem 3.2.2]) permet alors de supposer que l'on dispose d'un ensemble fini $S_{1} \subseteq \Omega_{k}$ contenant $S_{0}$ et pour toute $v \in S_{1}$, un zéro-cycle $z_{v}$ de degré 1 , supporté sur $X_{U} \otimes_{k} k_{v}$, tel que

$$
\sum_{v \in S_{1}} \operatorname{inv}_{v}\left(\left\langle\mathcal{A}, z_{v}\right\rangle\right)=0
$$

pour tout $\mathcal{A} \in B^{\prime}$. Notons $e$ l'exposant du groupe fini $B^{\prime}$. Pour toute place $v$, on a une écriture (évidente) $z_{v}=z_{v}^{+}-z_{v}^{-}$, où $z_{v}^{+}$et $z_{v}^{-}$sont des zéro-cycles effectifs. Écrivons

$$
\tilde{z}_{v}=z_{v}+n e z_{v}^{-} \text {, où } n=[M: k] .
$$

On obtient

$$
\sum_{v \in S_{1}} \operatorname{inv}_{v}\left(\left\langle\mathcal{A}, \tilde{z}_{v}\right\rangle\right)=0
$$

pour tout $\mathcal{A} \in B^{\prime}$. Choisissons un zéro-cycle effectif $\zeta$ sur $X_{U}$ qui est de degré $n$. Alors $\langle\mathcal{A}, e P\rangle=0$ pour $\mathcal{A} \in B^{\prime}$. Comme $\operatorname{deg} \tilde{z}_{v} \equiv 1 \bmod n e$ et $\operatorname{deg} e \zeta=n e$, on obtient - en ajoutant des multiples appropriés du zéro-cycle $e \zeta$ à chacun des $\tilde{z}_{v}$ - des cycles effectifs $\bar{z}_{v}$, tous du même degré $D \equiv 1(\bmod n e)$, tels que

$$
\sum_{v \in S_{1}} \operatorname{inv}_{v}\left(\left\langle\mathcal{A}, \bar{z}_{v}\right\rangle\right)=0
$$

pour tout $\mathcal{A} \in B^{\prime}$. En utilisant [9, lemma 6.2.1], on voit que l'on peut supposer que chacun de ces zéro-cycles effectifs $\bar{z}_{v}$ est somme de points fermés (sans multiplicités), dont les images sous $f: X_{U} \rightarrow U$ sont toutes différentes. On peut également supposer que pour tout point fermé $Q$ dans le support du zéro-cycle $\bar{z}_{v}$, l'extension de corps $k_{v}(f(P)) \subseteq k_{v}(P)$ est un isomorphisme: on remplace chaque point fermé $Q$ par un point $k_{v}(Q)$-rationnel de $X_{U} \otimes_{k} k_{v}$ suffisamment proche de $Q$.

Considérons maintenant les zéro-cycles (effectifs) $f\left(\bar{z}_{v}\right)$. Chacun de ces cycles est donné par un polynôme unitaire et séparable $G_{v}(t) \in k_{v}[t]$ de degré $D$, premier au produit $\prod_{i=1}^{s} F_{i}(t)$. Les fibres de $f$ au-dessus des racines de $G_{v}(t)$ admettent un point rationnel, pour $v \in S_{1}$. Il en est de même pour tout polynôme unitaire dans $k_{v}[t]$ suffisamment proche de $G_{v}(t)$ pour les topologies $v$-adiques sur l'espace des coefficients (Krasner).

Choisissons maintenant un polynôme unitaire, irréductible $G(t) \in k[t]$ de degré $D$ comme dans [8, theorem 3.1] ("l'astuce de Salberger"); pour le corps $L$ dans ce théorème, on prend une extension finie de $k$ qui contient $M$ et sur laquelle les $F_{i}(t)$ sont scindées. Pour $V$, on prend l'ensemble $T$ des places de $k$ qui scindent les $F_{i}(t)$ et qui sont totalement 
décomposées dans $L$. On peut choisir $G(t)$ suffisamment proche des $G_{v}(t)$ pour toute place $v \in S_{1}$ pour que les conditions suivantes soient satisfaites:

(i) $\mathrm{si}$

$$
N=k[t] /(G(t)), \theta=\bar{t} \in N,
$$

alors pour chacun des polynômes $F_{i}(t)$ (pour $1 \leqslant i \leqslant s$ ), il existe une place $w_{i}$ de $N$, telle que $F_{i}(\theta)$ soit une uniformisante dans la complétion $N_{w_{i}}$ et une unité dans $N_{w}$ si $w$ n'est pas au-dessus de $S_{1} \cup T$ (et $w \neq w_{i}$ );

(ii) pour $v \in S_{1}$ et toute place $w$ au-dessus de $v$, la fibre $X_{\theta}$ (définie sur $N$ ) admet un $N_{w}$-point $Q_{w}$, avec la somme $\sum_{w \mid v} Q_{w}$ suffisamment proche du zéro-cycle $\bar{z}_{v}$ pour que l'égalité

$$
\sum_{w \mid v} \operatorname{inv}_{v}\left(\operatorname{Cor}_{N_{w} / k_{v}}\left(\mathcal{A}\left(Q_{w}\right)\right)=\operatorname{inv}_{v}\left(\left\langle\mathcal{A}, \bar{z}_{v}\right\rangle\right)\right.
$$

vaille pour tout $\mathcal{A} \in B^{\prime}$.

Montrons maintenant que $X_{\theta}$ admet un $N_{w}$-point $Q_{w}$ pour toute place $w$ de $N$ qui n'est pas au-dessus de $S_{1}$. Si $w$ n'est pas non plus au-dessus de $T$ et n'est pas égale à l'une des $w_{i}$ pour $1 \leqslant i \leqslant s$, il est clair que $\left(\mathcal{X}_{\mathcal{U}}\right)_{\theta}$ admet un $\mathcal{O}_{N_{w}}$-point.

Si la place $w$ est au-dessus de $T$, alors $w$ est totalement décomposée dans l'extension $M N$ de $N$; il est donc évident que $X_{\theta}$ admet un $N_{w}$-point. Concentrons-nous donc sur le cas $w=w_{i}$ pour un $i \in\{1, \ldots, s\}$. On sait que

$$
\sum_{v \in S_{1}, w \mid v} \operatorname{inv}_{v}\left(\operatorname{Cor}_{N_{w} / k_{v}}\left(F_{i}(\theta), \chi\right)\right)=0
$$

et donc

$$
\sum_{v \notin S_{1}, w \mid v} \operatorname{inv}_{v}\left(\operatorname{Cor}_{N_{w} / k_{v}}\left(F_{i}(\theta), \chi\right)\right)=0 .
$$

Or, si $w$ n'est pas au-dessus de $S_{1} \cup T$ et $w \neq w_{i}$, alors $F_{i}(\theta)$ est une unité dans $N_{w}$, et si $w$ est au-dessus de $T$, alors $w$ est totalement décomposée dans l'extension $M N$ de $N$. Dans les deux cas, $\operatorname{inv}_{w}\left(F_{i}(\theta), \chi\right)=0$. Il en résulte que

$$
\operatorname{inv}_{w_{i}}\left(F_{i}(\theta), \chi\right)=0
$$

pour tout caractère $\chi$ de $M / k$. Comme $F_{i}(\theta) \in N$ est une uniformisante dans $N_{w_{i}}$ et l'extension $M / k$ est abélienne, on voit que $w_{i}$ est totalement décomposée dans l'extension $M N$ de $N$. La fibre $X_{\theta}$ admet donc un $N_{w_{i}}$-point.

Exactement comme dans la preuve du Théorème $1 \cdot 2$, on démontre que $\operatorname{inv}_{w}\left(\mathcal{A}\left(Q_{w}\right)\right)=0$ pour tout $\mathcal{A} \in B$ et toute $w \in \Omega_{N}$ non au-dessus de $S_{1}$.

On obtient donc l'égalité

$$
\sum_{w \in \Omega_{N}} \operatorname{inv}_{w}\left(\mathcal{A}\left(Q_{w}\right)\right)=0
$$

La flèche naturelle

$$
\operatorname{Br}\left(T^{c}\right) / \mathrm{Br}_{0}\left(T^{c}\right) \longrightarrow \operatorname{Br}\left(T_{N}^{c}\right) / \operatorname{Br}\left(T_{N}^{c}\right)
$$

est un isomorphisme, car $M \cap N=k$. L'image de $B$ engendre donc bien le quotient $\operatorname{Br}\left(X_{\theta}\right) / \operatorname{Br}_{0}\left(X_{\theta}\right)$. Une application du résultat de Sansuc [16, corollaire 8.7] à la fibre $X_{\theta}$ donne l'existence d'un $N$-point sur la fibre $X_{\theta}$. Comme $[N: k] \equiv 1(\bmod n e)$, ceci implique bien l'existence d'un zéro-cycle de degré 1 sur $X$. 


\section{Une remarque sur l'hypothèse de Schinzel}

On présente dans ce dernier paragraphe une remarque sur l'hypothèse de Schinzel qui permet de l'utiliser de façon plus souple. Il s'agit du choix d'un paramètre de Schinzel $\lambda$, comme dans $\left[9\right.$, hypothèse $\left.\left(\mathrm{H}_{1}\right)\right]$, avec la condition supplémentaire que cette valeur de $\lambda$ appartienne à un sous-ensemble hilbertien donné de $k$. Que ceci devrait être possible semble avoir été suggéré pour la première fois dans [19].

\subsection{Sur la topologie d'un ensemble Hilbertien}

Il nous faut un résultat préliminaire sur la topologie d'un ensemble hilbertien; pour cette dernière notion, voir par exemple [11].

Proposition 6·1. Soit $k$ un corps de nombres. Soit $V$ une $k$-variété géométriquement intègre. Soit $\rho: X \rightarrow V$ un revêtement étale connexe et soit $P \in V(k)$ tel que la fibre de $\rho$ en $P$ soit connexe. Soit $S \subseteq \Omega_{k}$ un ensemble fini de places de $k$. Alors il existe un ensemble fini $T \subseteq \Omega_{k}^{<\infty}$ avec $S \cap T=\varnothing$ et un voisinage $N$ de $P$ dans $\prod_{v \in T} V\left(k_{v}\right)$ dans la topologie naturelle tel que pour tout $Q \in N(k)$, la fibre $\rho^{-1}(Q)$ soit connexe.

Démonstration. On agrandissant $S$ si nécessaire, on peut supposer que la situation s'étend sur $\mathcal{O}_{k, S}$. Partons donc d'un morphisme dominant $\pi: \mathcal{V} \rightarrow \operatorname{Spec}\left(\mathcal{O}_{k, S}\right)$ dont la fibre générique est une $k$-variété géométriquement intègre, d'un revêtement étale connexe $\rho: \mathcal{X} \rightarrow \mathcal{V}$ de fibre générique intègre et d'un point $P \in \mathcal{V}\left(\mathcal{O}_{k, S}\right)$ tel que la fibre de $\rho$ en $P$ soit connexe. On peut supposer également que $\rho$ est Galoisien de groupe $G$.

Démontrons d'abord que pour tout élément $g \in G$, il existe une place finie $v_{g} \in \Omega_{k}^{<\infty} \backslash S$ telle que Frob $_{v_{g}} P$ est conjugué à $g$. Fixons $g \in G$. Écrivons $\mathcal{X}^{\prime}=\mathcal{X} /\langle g\rangle$, le quotient de $\mathcal{X}$ par l'action de $\langle g\rangle$, qui est un revêtement étale de $\mathcal{V}$. Considérons le diagramme commutatif suivant, dont les carrés sont Cartésiens:

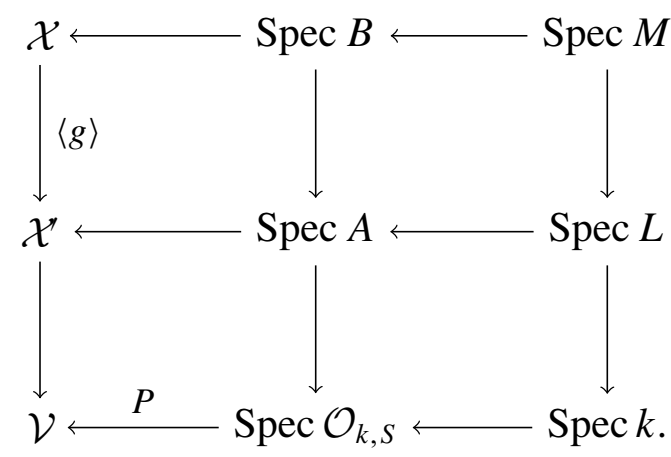

La fibre du morphisme $\rho: \mathcal{X} \rightarrow \mathcal{V}$ au-dessus du $k$-point $P$ est connexe. Les $k$-algèbres $L$ et $M$ sont donc bien des corps et l'extension $M / L$ est cyclique, de groupe $\langle g\rangle$. Ceci implique que $A / \mathcal{O}_{k, S}$ est une extension finie d'anneaux de Dedekind.

Donc [12, proposition 2.2.1] s'applique: il existe une place $v_{g}$ de $k$ en dehors de $S$ et une place $w$ de $L$ au-dessus de $v_{g}$ qui est de degré 1 et qui est inerte dans $M$. Par construction, Frob $_{v_{g}} P$ est conjugué à $g$.

Ceci étant établi, choisissons pour tout élément $g \in G$ une telle place $v_{g}$ et prenons $T=\left\{v_{g} \mid g \in G\right\}$. Prenons pour $\mathcal{N}$ l'ensemble des $k$-points de $\mathcal{V}$ qui sont entiers en les places de $T$ et qui, pour tout $g \in G$, ont la même réduction (comme $\kappa\left(v_{g}\right)$-point) que $P$. Il suffit de montrer que $\rho^{-1}(Q)$ est connexe pour tout $Q \in \mathcal{N}$. Ceci équivaut à dire que le groupe de Galois $\operatorname{Gal}(\bar{k} / k)$ se surjecte sur $G$ à travers un relèvement du morphisme composé

$$
\operatorname{Spec} \bar{k} \longrightarrow \operatorname{Spec} k \stackrel{Q}{\longrightarrow} \mathcal{V}
$$

à $\mathcal{X}$. Par construction des places $v_{g}$, cette dernière assertion est satisfaite. 
Corollaire 6.2. Si on admet l'hypothèse de Schinzel [9, hypothèse $(\mathrm{H})]$ sur $\mathbf{Q}$, alors la version $\left[\mathbf{9}\right.$, hypothèse $\left.\left(\mathrm{H}_{1}\right)\right]$ de cette hypothèse vaut sur tout corps de nombres $k$, avec la condition supplémentaire que l'on peut choisir le paramètre $\lambda$ dans un ensemble hilbertien $H \subseteq k=\mathbb{A}_{k}^{1}(k)$ donné.

Démonstration. Partons de polynômes $P_{i}(t)$ pour $1 \leqslant i \leqslant n$ et d'éléments $\lambda_{v} \in k_{v}$ pour $v \in S$, où $S \subseteq \Omega_{k}$ est fini, comme dans [9, hypothèse $\left.\left(\mathrm{H}_{1}\right)\right]$. En agrandissant $S$ si nécessaire, on peut supposer que l'ensemble hilbertien $H$ est associé à un revêtement étale de $\mathcal{O}_{k, S}$-schémas $\rho: \mathcal{X} \rightarrow \mathbb{A}_{\mathcal{O}_{k, S}}^{1}$, comme dans la Proposition 6·1.

Choisissons un $k$-point $P$ de coordonnée $\mu$ tel que la fibre $\rho^{-1}(P)$ soit connexe. On trouve un ensemble fini $T \subseteq \Omega_{k}^{<\infty}$ avec $S \cap T=\varnothing$ et un voisinage $\mathcal{N}$ de $P$ dans la topologie induite par $T$ tel que $\mathcal{N} \subseteq H$. Prenons $\lambda_{v}=\mu \in k_{v}$ pour $v \in T$. En appliquant [9, hypothèse $\left.\left(\mathrm{H}_{1}\right)\right]$ aux polynômes $P_{i}(t)$ pour $1 \leqslant i \leqslant n$ et aux $\lambda_{v}$ pour $v \in S \cup T$, on obtient maintenant le résultat voulu.

\subsection{Exemple d'application}

L'intérêt du Corollaire 6.2 ci-dessus dans le contexte de la méthode des fibrations vient de [12, théorème 3.5.1]. Voici un simple exemple d'application dans ce sens, qui généralise $[8$, theorem 1.1.e]:

Corollaire 6.3. Soit $X$ une $k$-variété projective et lisse, munie d'un morphisme dominant $f: X \rightarrow \mathbb{P}_{k}^{1}$, telle que la condition suivante soit satisfaite: pour tout point fermé $P$ de $\mathbb{P}_{k}^{1}$, la fibre $X_{P}$ a une composante $Y_{P}$ de multiplicité 1 telle que la clôture algébrique de $k(P)$ dans le corps de fonctions de $Y_{P}$ soit une extension abélienne de $k(P)$.

Supposons que le principe de Hasse et l'approximation faible valent pour les fibres de $f$ au-dessus d'un ensemble hilbertien de $\mathbb{P}_{k}^{1}$. Admettons l'hypothèse de Schinzel. Alors $X(k)$ est dense dans $X\left(\mathbf{A}_{k}\right)^{\mathrm{Br}_{\mathrm{ver}}(X)}$.

Rappelons que le groupe de Brauer vertical $\operatorname{Br}_{\text {vert }}(X)$ est le sous-groupe de $\operatorname{Br}(X)$ qui consiste des éléments dont la restriction à $\operatorname{Br}\left(X_{\eta}\right)$ provient de $\operatorname{Br}(\eta)$.

La validité de cet énoncé résulte immédiatement du Corollaire 6.2 et de la preuve de [8, theorem 1.1]. Voici un exemple d'application concrète de ce résultat.

Proposition 6.4. Soit X un modèle projectif et lisse d'une k-variété affine donné par une équation de la forme

$$
x^{2}-a(t) y^{2}=P(t, z),
$$

où le polynôme $P(t, z)$ est irréductible de degré 4 vu comme élément de $k(t)[z]$ et a la propriété que $P\left(t_{0}, z\right)$ ne s'annule pas identiquement si $t_{0}$ est une racine de a $(t)$. Admettons l'hypothèse de Schinzel. Alors $X(k)$ est dense dans $X\left(\mathbf{A}_{k}\right)^{\operatorname{Br}_{\text {vert }}(X)}$.

Démonstration. La variété $X$ est fibrée sur $\mathbb{P}_{k}^{1}$ via la projection sur $t$. Il est clair que l'hypothèse sur les "mauvaises" fibres dans le Corollaire $6 \cdot 3$ est satisfaite sous les conditions de l'énoncé. L'ensemble des valeurs $s \in k$ ayant la propriété que le polynôme $P(s, z) \in k[z]$ reste irréductible est un ensemble Hilbertien dans $\mathbb{A}_{k}^{1}(k)$. Pour une telle valeur de $s$, la surface de Châtelet donnée par l'équation affine $x^{2}-a(s) y^{2}=P(s, z)$ satisfait au principe de Hasse et à l'approximation faible [7]. Ceci suffit pour appliquer le Corollaire 6·3. 


\section{Remerciements}

Je voudrais rémercier Jean-Louis Colliot-Thélène pour son aide, ses encouragements et sa patience pendant l'élaboration de ce texte. Je remercie également Olivier Wittenberg pour des discussions utiles, notamment sur la Proposition 6·1.

L'auteur est aspirant du FWO (Fonds de la Recherche Scientifique - Flandres). Pendant la préparation de ce texte, il a également bénéficié du soutien du Centre Interfacultaire Bernoulli (EPFL) et du Hausdorff Institute for Mathematics (Bonn).

\section{REFERENCES}

[1] T. D. BRowning, D. R. HEATH-BROWN. Quadratic polynomials represented by norm forms. Geom. Funct. Anal. 22 (2012), 1124-1190.

[2] J.-L. Colliot-Thélène. Groupe de Brauer non ramifié d'espaces homogènes de tores. J. Théor. Nombres Bordeaux. 26 (2014), 69-83.

[3] J.-L. Colliot-ThÉlÈnE. Variétés presque rationnelles, leurs points rationnels et leurs dégénérescences. Paru dans Arithmetic Geometry (CIME 2007), (Springer LNM 2009) (2011), $1-44$.

[4] J.-L. Colliot-Thélène, D. Harari and A. N. Skorobogatov. Valeurs d'un polynôme à une variable représentées par une norme. Paru dans le volume Number theory and algebraic geometry. London Math. Soc. Lecture Note Ser. vol. 303 (Cambridge University Press, Cambridge, 2003), 69-89.

[5] J.-L. Colliot-ThÉLÈne and J.-J. SAnsuc. La descente sur les variétés rationnelles. II. Duke Math. J. 54 (2) (1987), 375-492.

[6] J.-L. Colliot-Thélène and J.-J. SAnsuC. Principal homogeneous spaces under flasque tori: applications. J. Algebra 106 (1) (1987), 148-205.

[7] J.-L. Colliot-ThÉlÈne, J.-J. SAnSUC and P. SwinnerTon-Dyer. Intersections of two quadrics and Châtelet surfaces. I. J. Reine Angew. Math. 373 (1987), 37-107.

[8] J.-L. Colliot-Thélène, A. N. Skorobogatov and P. Swinnerton-Dyer. Rational points and zero-cycles on fibred varieties: Schinzel's hypothesis and Salberger's device. J. Reine Angew. Math. 495 (1998), 1-28.

[9] J.-L. Colliot-ThÉlÈne and P. Swinnerton-Dyer. Hasse principle and weak ap-proximation for pencils of Severi-Brauer and similar varieties. J. Reine Angew. Math. 453 (1994), 49-112.

[10] U. Derenthal, A. Smeets and D. Wei. Universal torsors and values of quadratic polynomials represented by norms. Math. Ann. published online (2014).

[11] T. EKEDAhl. An effective version of Hilbert's irreducibility theorem. Séminaire de théorie des nombres de Paris 1988-1989, éd. C. Goldstein. Progr. Math. 91 (Birkhäuser, Boston, 1990), 241248.

[12] D. Harari. Méthode des fibrations et obstructions de Manin. Duke Math. J. 75 (1994), 221-260.

[13] D. R. HeAth-Brown and A. Skorobogatov. Rational solutions of certain equations involving norms. Acta Math. 189 (2) (2002), 161-177.

[14] Y. Harpaz, A. Skorobogatov and O. Wittenberg. The Hardy-Littlewood conjecture and rational points. Compositio Math. published online (2014).

[15] Y. LiAnG. Towards the Brauer-Manin obstruction on varieties fibred over the projective line. J. Algebra 413 (2014), 50-71.

[16] J.-J. SANSUC. Groupe de Brauer et arithmétique des groupes algébriques linéaires sur un corps de nombres. J. Reine Angew. Math. 327 (1981), 12-80.

[17] D. Schindler and A. N. Skorobogatov. Norms as products of linear polynomials. J. London Math. Soc. 89 (2014), 559-580.

[18] A. Skorobogatov. Descent on fibrations over the projective line. Amer. J. Math. 118 (1996), 905923.

[19] P. SWINNERTON-DYER. Lettre à O. Wittenberg du 10 Janvier 2005.

[20] A. VÁRILly-AlVARADO and B. ViRAY. Higher dimensional analogues of Châtelet surfaces. Bull. London Math. Soc. 44 (2012), 125-135.

[21] D. WEI. On the equation $N_{K / k}(\Xi)=P(t)$. Proc. London Math. Soc. published online (2014). 